\title{
Use of light wand as an adjunct during intubation of patient with large epiglottic cyst
}

\author{
Jun Hyun Kim, Kyung Woo Kim, Jina Park, Kyung Tae Kim, and Jang Su Park \\ Department of Anesthesiology and Pain Medicine, Ilsan Paik Hospital, Inje University School of Medicine, Goyang, Korea
}

Epiglottic cysts sometimes compromise a patient's airway, and may make airway management difficult. We report a novel method of intubating a patient with a large epiglottic cyst, using direct laryngoscopy and a light wand.

A 53-year-old man with hoarseness was admitted to the hospital. He complained of a mild cough and sore throat for 2 days, without dyspnea. General anesthesia for microscopic laser surgery was planned. Neck computed tomography revealed a nearly non-enhancing cyst, $2.7 \times 1.8 \times 3.3 \mathrm{~cm}$, located on the right prelingual surface of the epiglottis (Figs. 1A and 1B).

In addition to standard monitoring and preparations, we prepared laryngeal mask airways, adult and pediatric fiberoptic bronchoscopes, and an emergency cricothyrotomy kit at the bedside. Two attending anesthesiologists and one resident were present in the operating room. After preoxygenation, propofol $120 \mathrm{mg}$ was given. The patient became unconscious, and we confirmed there was no difficulty with mask ventilation. We then administered rocuronium $25 \mathrm{mg}$ intravenously. After 4 minutes, we attempted intubation with a Macintosh blade no. 3 and 5.0 laser tube (Laser-Flex ${ }^{\circledR}$ Tracheal Tube, Mallinckrodt, St. Louis, MO, USA) without stylet. We carefully placed the tip of the Macintosh blade at the vallecula, avoiding direct pressure on the cyst. After that, an assistant held the handle of the laryngoscope steady. The intubating anesthesiologist then held the hockey stick-shaped light wand in the left hand and used it both for leverage and as a light source. Gently elevating both the epiglottis and cyst with the light wand in the upper left direction revealed the lower third of the glottis with sufficient light. We then intubated the patient.

After intubation, we began mechanical ventilation with air.
Tidal volume was set to $500 \mathrm{ml}$, respiration rate was 12 per minute, and peak airway pressure was $26 \mathrm{cmH}_{2} \mathrm{O}$. Anesthesia was maintained with sevoflurane $1.5-2.0 \mathrm{vol} \%$ and remifentanil continuous infusion of $0.1-2.0 \mathrm{mcg} / \mathrm{kg} / \mathrm{min}$. Oxygen saturation was maintained at a minimum of $95 \%$ throughout the surgery and in the recovery room. The patient recovered and was discharged after 1 day without any adverse events.

Most epiglottic cysts are located on the lingual surface or vallecula, which makes intubation difficult, when a cyst is large. For the treatment of epiglottic cysts, simple aspiration of the cyst results in frequent recurrence, so complete removal is recommended [1]. For this removal, laser surgery seems to be superior to cold instrument surgery, in many respects.

During laser surgery, the use of laser resistant endotracheal tubes is recommended to avoid catastrophic airway fires. There are several choices for laser resistant endotracheal tubes, including wrapping metal foil around conventional tubes, Xomed Laser-Shield (Laser-Shield ${ }^{\circledR}$ II Endotracheal Tube, Medtronic Xomed, Jacksonville, FL, USA), and Mallinckrodt Laser-Flex. Inevitably, methods or tubes such as these are accompanied by an increase in the wall thickness of the tubes. With the LaserFlex, for example, the outer diameter (OD) of the tubes is at least $2.5 \mathrm{~mm}$ greater than the internal diameter (ID). This number is considerable in comparison to the thickness of conventional tubes, which is $1.3 \mathrm{~mm}$ at minimum.

In our case, when intubation was attempted, the epiglottic cyst blocked the light from the laryngoscope, so structures behind the cyst were not visible (Fig. 1C). To circumvent this problem, there are several possible options. First, we could use a Miller blade or a Magill blade. Holding the epiglottis from the

Corresponding author: Jun Hyun Kim, M.D., Department of Anesthesiology and Pain Medicine, Ilsan Paik Hospital, Inje University School of Medicine, 2240, Daewha-dong, Ilsanseo-gu, Goyang 411-706, Korea. Tel: 82-31-910-7160, Fax: 82-31-910-7184, E-mail: urdesire@hanmail.net (c) This is an open-access article distributed under the terms of the Creative Commons Attribution Non-Commercial License (http:// creativecommons.org/licenses/by-nc/3.0/), which permits unrestricted non-commercial use, distribution, and reproduction in any medium, provided the original work is properly cited. 

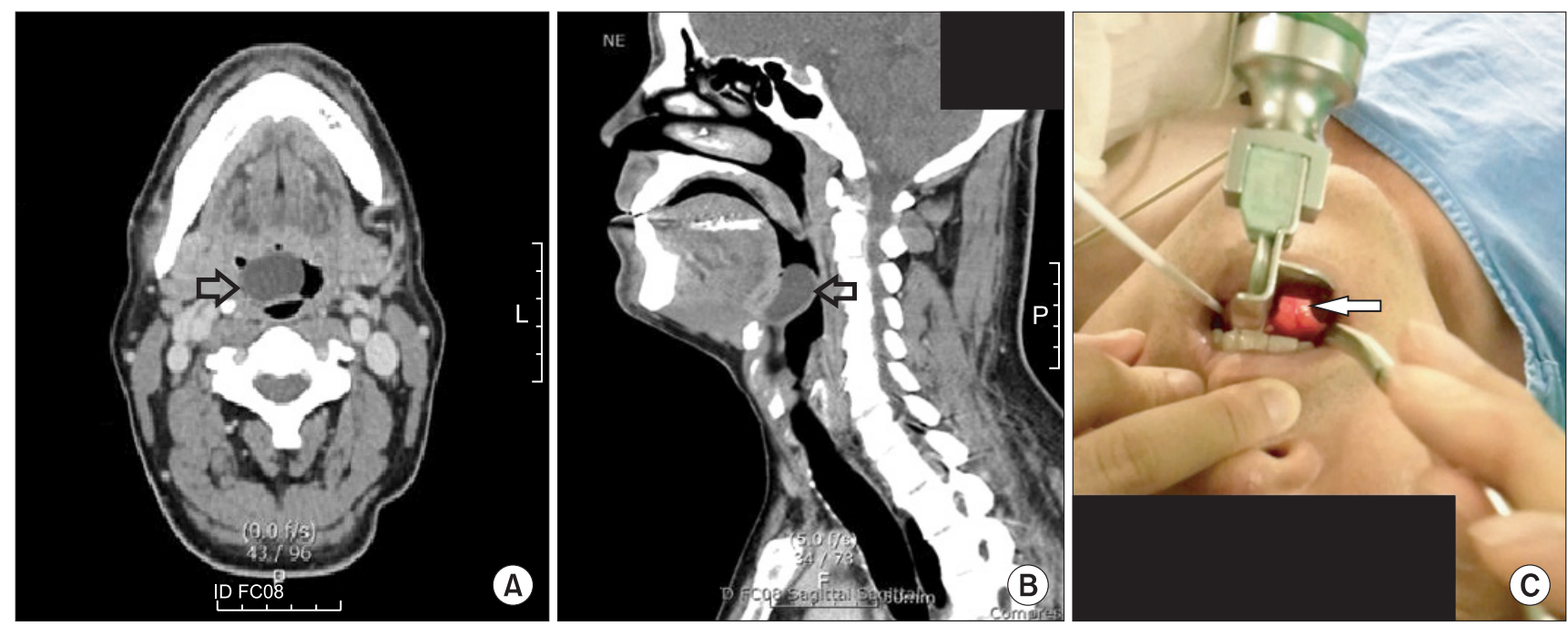

Fig. 1. Neck computed tomography (CT) revealed a nearly non-enhancing cyst in the right pre-epiglottic space with concomitant mild airway obstruction ([A] Transverse and [B] Sagittal views). (C) This image shows direct laryngoscope, light wand and laser tube in position before the leverage. Arrow is indicating epiglottic cyst blocking the light.

lower surface, together with the cyst, may allow direct visualization of the vocal cords without blockage of the light. However, considering the size of the cyst, this maneuver might fail. In addition pressure of the blade may rupture the cyst and cause pulmonary aspiration of its contents. Second, we could aspirate the contents of the cyst and then try the conventional intubation method. If the mass was not entirely cystic, or lobulated, the chances of success would be slim. Furthermore, attempting aspiration without first securing the airway may significantly increase the risk of pulmonary aspiration of cyst contents. Lastly, we could choose larger diameter tubes to use a light wand, intubating stylet, or fiberoptic bronchoscope.

For airway surgery, though, it is rational to choose the smallest tubes possible to facilitate the surgery. We chose an ID 5.0 Laser tube, the OD of which is equivalent to the OD of an ID 5.5 conventional tube. The OD of an adult fiberoptic bronchoscope used for awake intubation is $5.0 \mathrm{~mm}$, the same as the ID of the Laser tube. The OD of a light wand is $5.55 \mathrm{~mm}$, still larger than the ID of the tube. The OD of the intubating stylet was $4.65 \mathrm{~mm}$, but due to small differences between the ID of the tube and the OD of the stylet, using it was impossible. In patients with more difficult airways, it may be rational to choose a Laser-Flex tube with a bigger ID and use a stylet in it, but this also may be difficult because of the dimmed light caused by the cyst. A light wand may still be difficult to use because of the large OD of a light wand for an ID 6.0 Laser tube. The difference in diameter between the two is only $0.45 \mathrm{~mm}$. Laser tubes are rigid and difficult to use with a fiberoptic bronchoscope. In addition, to use an adult bronchoscope, one must use a tube with an ID larger than $6.0 \mathrm{~mm}$. A McCoy blade can be helpful in some situations. In our case it might be effective, but considering the size and the location of the cyst, even elevating the epiglottis might not make it possible to move the cyst away from the light source. In actuality, levering of the epiglottis in the ventral and cranial direction would move the cyst closer to the light source and might make the view dimmer.

Using a fiberoptic bronchoscope or light wand in the presence of a large epiglottic cyst is also a difficulty, because of the mass blocking the passage. Recently, Choi et al. [2] reported a case of intubation of a patient with a large glottic mass. In their report, introducing a flexible bronchoscope was difficult and ultimately not possible, in spite of attempts at guidance and visualization with the GlideScope ${ }^{\circledR}$ (Verathon Inc., Bothell, WA, USA) video laryngoscope. The authors did not report the size of the mass in their case, but the picture included in their report shows similarity to our case.

Epiglottic cysts poses great challenges for anesthesiologists in some cases. Our method can be a relatively easy, less invasive, simple method dealing with some large epiglottic cysts.

\section{References}

1. Su CY, Hsu JL. Transoral laser marsupialization of epiglottic cysts. Laryngoscope 2007; 117: 1153-4.

2. Choi GS, Park SI, Lee EH, Yoon SH. Awake Glidescope ${ }^{\circledR}$ intubation in a patient with a huge and fixed supraglottic mass -A case report-. Korean J Anesthesiol 2010; 59 Suppl: S26-9. 